\title{
Daily Incremental Lines in Sika Deer (Cervus nippon) Dentine
}

\author{
Yasuko IINUMA $^{1)}$, Masatsugu SUZUKI ${ }^{1)}$, Mayumi YOKOYAMA ${ }^{2)}$, Yuka TANAKA-NAKAMURA ${ }^{1)}$ and \\ Noriyuki OHTAISHI ${ }^{1)}$ \\ ${ }^{1)}$ Laboratory of Wildlife Biology, Graduate School of Veterinary Medicine, HokkaidoUniversity, Kita-ku, Sapporo 060-0818 and \\ ${ }^{2)}$ Division of Environmental Management, Museum of Nature and Human Activities, Sanda 669-1546, Japan
}

(Received 5 February 2002/Accepted 7 May 2002)

ABSTRACT. This work was designed to observe the dentine incremental lines of the sika deer (Cervus nippon) fawns and to investigate their periodicity using the chronological labeling method with fluorochromes. The incremental lines were observed in decalcified specimens stained by Bodian's silver technique, and the fluorescence-labeled lines were observed in undecalcified and ground specimens. In the silver stained specimens, there were two types of lines, deeply stained thick lines and faintly stained minute regular incremental lines. The intervals and staining intensities of the deeply stained thick lines were very similar to those of the fluorescence-labeled lines in the ground specimens obtained from the same tooth, and hence, it appeared that the both lines were identical. The number of minute incremental lines between the deeply stained thick lines was the same as that of days between the time when each fluorescent labeling injection was made. Therefore, it seemed that each minute incremental line was formed each day. The possibility of age estimation in days using diurnal dentine increments was discussed.

KEY WORDS: Cervus nippon, circadian rhythm, dentine, fluorescent labeling, incremental line.

J. Vet. Med. Sci. 64(9): 791-795, 2002

Periodic growth incremental lines are found universally in the dental tissues of animals [7, 10,15]. The formation of these incremental lines is a visible result of the morphological change of tooth structure as the teeth respond to changes in the physiological parameters of an organism [7]. Annual layers in the cementum are the most familiar dental increments, commonly used for age estimation in many mammals [2]. Similar incremental lines also appear in the dentine and enamel, formed in a density higher than in the cementum. Previous studies have demonstrated that these dentine or enamel increments reflect circalunarl or circadian rhythms in various animals $[1,9,10,16,18]$. In wild Altai gray birch mice (Sicista Pseudonapaea), the ecological relevance of these increments has also been investigated, and the sexual differences in their life history were revealed by observation of the incremental lines in the incisor dentine [8]. However, in large mammals, few reports are available on the dentine increments.

Estimation of age in days or months, if available, would make it possible to clarify more in detail the wild life history of animals, such as Altai gray birch mice. In this sense, the examination and the resultant better understanding of dental increments would contribute to further betterment of wildlife management.

Therefore, the present study was performed to examine the incremental lines in the dentine of the sika deer (Cervus nippon), one of the most important game animals and a target species for wildlife management in Japan [4], followed by discussion of the possibility of month-age or day-age estimation for this species.

\section{MATERIALS AND METHODS}

Sample collection: Six sika deer fawns were obtained from Satomigaoka Game Farm of Ashoro district (143E, $43 \mathrm{~N}$ ) in eastern Hokkaido. Under anesthesia with a combination of xylazine hydrochloride $(1.2 \mathrm{mg} / \mathrm{kg})$ and ketamine hydrochloride $(1.5 \mathrm{mg} / \mathrm{kg})$ by means of a hand-made blowpipe, each deer was given an alternate intramuscular injection of oxytetracycline (Wako Pure Chemicals, Inc., Osaka, Japan) and calcein (Wako) at a dose level of $40 \mathrm{mg} / \mathrm{kg}$ and 6 $\mathrm{mg} / \mathrm{kg}$ body weight, respectively. Injections of these vital stains were given at 13-19 day intervals between August and October in 1998. The detailed injection histories are shown in Fig. 1 .

These deer were slaughtered on day 14 or 15 of December in 1998 by exsanguination under anesthesia. Among the teeth, the lower first incisor of each deer was selected for observation, since it was so simple in shape as to discriminate easily its neck from the other part. The incisor of each deer was removed and fixed in $10 \%$ buffered formalin for two weeks, and then stored in $70 \%$ ethanol until observation. It was embedded in methyl methacrylate resin to be polymerized at $40^{\circ} \mathrm{C}$ for 5 days.

From each incisor, two types of specimens, undecalcified and ground, and decalcified and stained, were prepared for the observation of fluorescence-labeled lines and minute incremental lines, respectively.

Undecalcified and ground specimens: Using a diamondedged saw, a $300 \mu \mathrm{m}$-thick cross section of the incisor neck (Fig. 2) was prepared from each polymerized tooth. The section was ground and polished to be $150-200 \mu \mathrm{m}$ in thickness using an ML-150P lapping unit (Maruto, Inc., Tokyo, Japan). The polished specimen was mounted on a microscope slide with distilled water and observed under an LSM410 confocal laser scanning microscope (CLSM) (Carl zeiss, Inc., Jena, Germany). The excitation wavelength was set at 364 and $488 \mathrm{~nm}$ for oxytetracycline and calcein, 


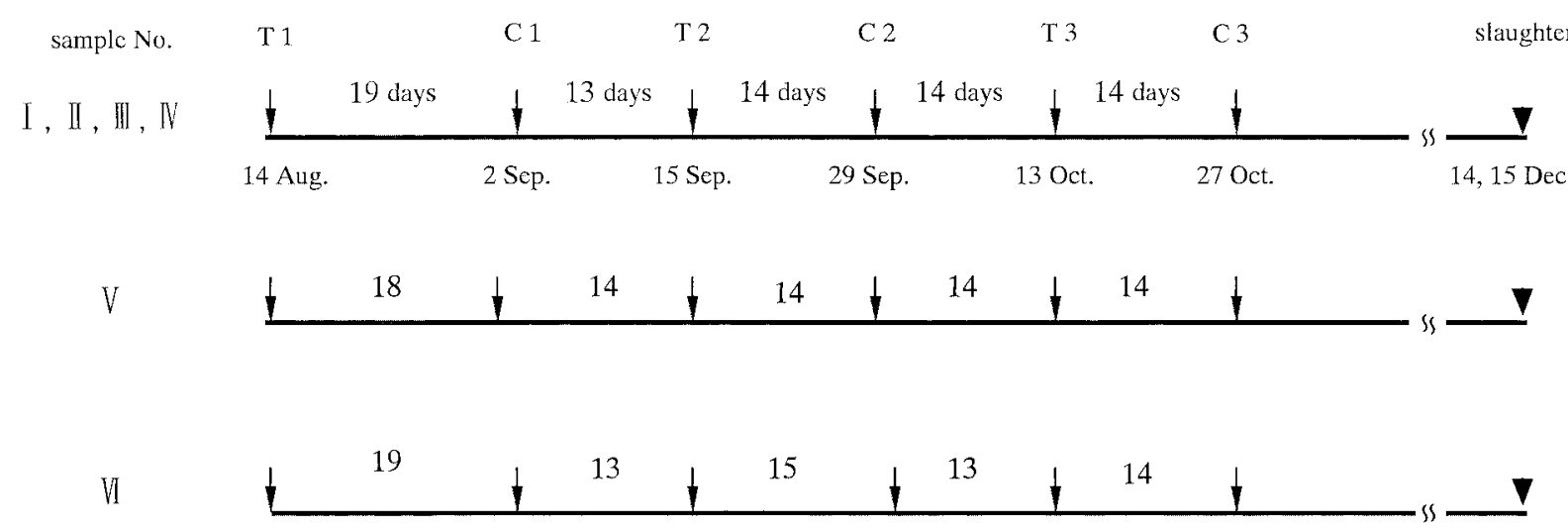

Fig. 1. Injection histories for the chronological labeling method. T1: the first injection of tetracycline, $\mathrm{C} 1$ : the first injection of $\mathrm{c}$ alcein, T2: the second injection of tetracycline, C2: the second injection of calcein, T3: the third injection of tetracycline, C3: thethird injection of calcein.

respectively, and the fluorescence was detected through a $570 \mathrm{~nm}$ long pass filter and a 515-565 nm band pass filter, respectively.

Decalcified and stained specimens: An approximately $2-$ $3 \mathrm{~mm}$ thick cross section was made from a part adjacent to the foregoing undecalcified specimen (Fig. 2). After removal of polymerizing resin by methyl methacrylate monomer, the section was decalcified in Plank-Rychlo solution for two days. Washed with tap water and embedded in O. C. T. compound (Miles Inc., Elkhart, U. S. A.), the section was further cross-sectioned into $16 \mu \mathrm{m}$ in thickness in a cryostat, mounted on a glass slide, and dried at room temperature.

According to Bodian's silver technique, these thin sections were soaked in distilled water (DW), stained with $2.5 \%$ protargol (silver protein: E. Merck Darmstadt, Frankfurter, Germany) solution containing copper chips (5 g to $100 \mathrm{~m} l$ staining solution) for $30 \mathrm{hr}$ at $40^{\circ} \mathrm{C}$, and then rinsed again with DW. The reduction of silver in sections was accomplished with a solution containing hydroquinone and formalin for $10 \mathrm{~min}$. After being washed thoroughly with $\mathrm{DW}$, the sections were treated with a solution of $0.2 \%$ gold chloride for $40 \mathrm{~min}$, and then rinsed again with DW. The color balance of the stained sections was modulated with $2 \%$ oxalic acid solution for $5 \mathrm{~min}$. After these treatments, the sections were rinsed with DW and dipped in a solution of $5 \%$ sodium thiosulfate for $15 \mathrm{~min}$ in order to remove residual silver salt [6]. The sections were finally washed thoroughly, dehydrated, mounted in bioleit, and observed under transmitted light.

\section{RESULTS}

Under the CLSM, every sample of undecalcified and ground sections revealed tetracycline-labeled lines and calcein-labeled lines. The numbers of both lines were in accord with the frequencies of injections. Calcein produced more strongly and more clearly stained lines than did tetra-

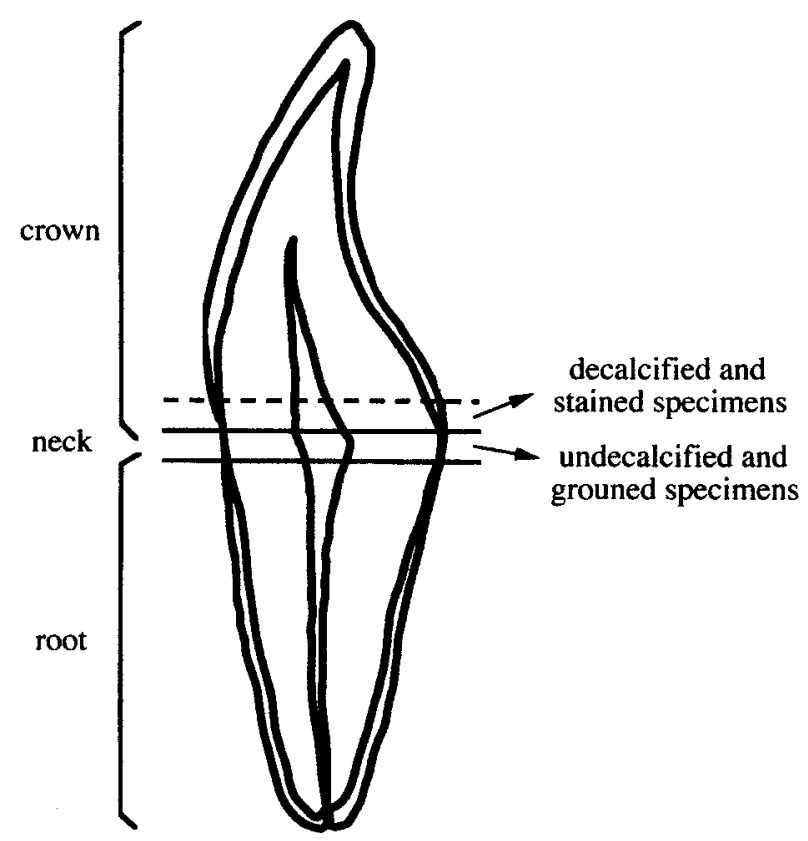

Fig. 2. Diagram of incisor (longitudinal plane) showing locational relation of the undecalcified and ground specimen and the decalcified and stained specimen.

cycline (Fig. 3a). The intervals and staining intensities of both fluorescent lines tended to be narrowed and weakened toward the dentine's inner side.

In the decalcified sections stained by Bodian's silver technique, there were two types of argyrophilic lines, faintly stained minute regular line, and deeply stained slightly thick line (Figs. 3b and 4). The interval, intensity, and running pattern of these deeply stained argyrophilic lines were very similar to those of the fluorescence-labeled lines in the ground section (Fig. 3).

The number of minute regular lines between successive deeply stained lines was countable in the three deer. From 

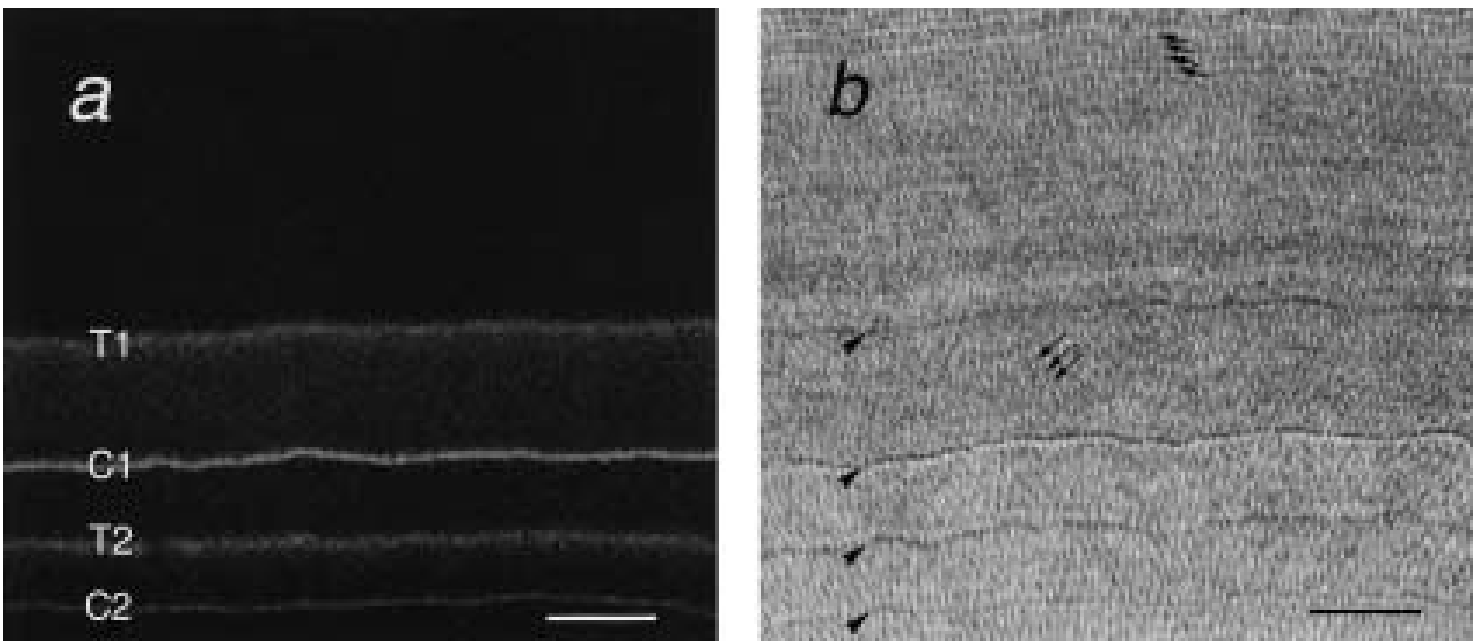

Fig. 3. Comparison of ground and stained specimens collected from the same tooth. Scale bar $=50 \mu \mathrm{m}$ a: ground cross-section of the cervical part of incisor. Fluorescence-labeled lines: the number of lines is in accord with the injection times. Calcein labels the lines more strongly and more clearly than does tetracycline. The intervals and staining intensities of both fluorescent line s tend to be narrowed and weakened toward the pulp side of the dentine (bottom of the figure). b: decalcified cross-section treated by Bodian's silver technique and obtained from a part adjacent to a. The two types of lines, faintly stained minute regular lines (arrows) and deeply stained thick lines (arrow heads), are seen. The intervals and staining intensities of these deeply stained thick lines are almost the same as those of the fluorescence-labeled lines in a. The faintly stained minute lines become unclear toward the pulp. T1: labeled line by the first tetracycline injection. C1: labeled line by the first calcein injection. T2 labeled line by the second tetracycline injection. $\mathrm{C} 2$ : labeled line by the second calcein injection.

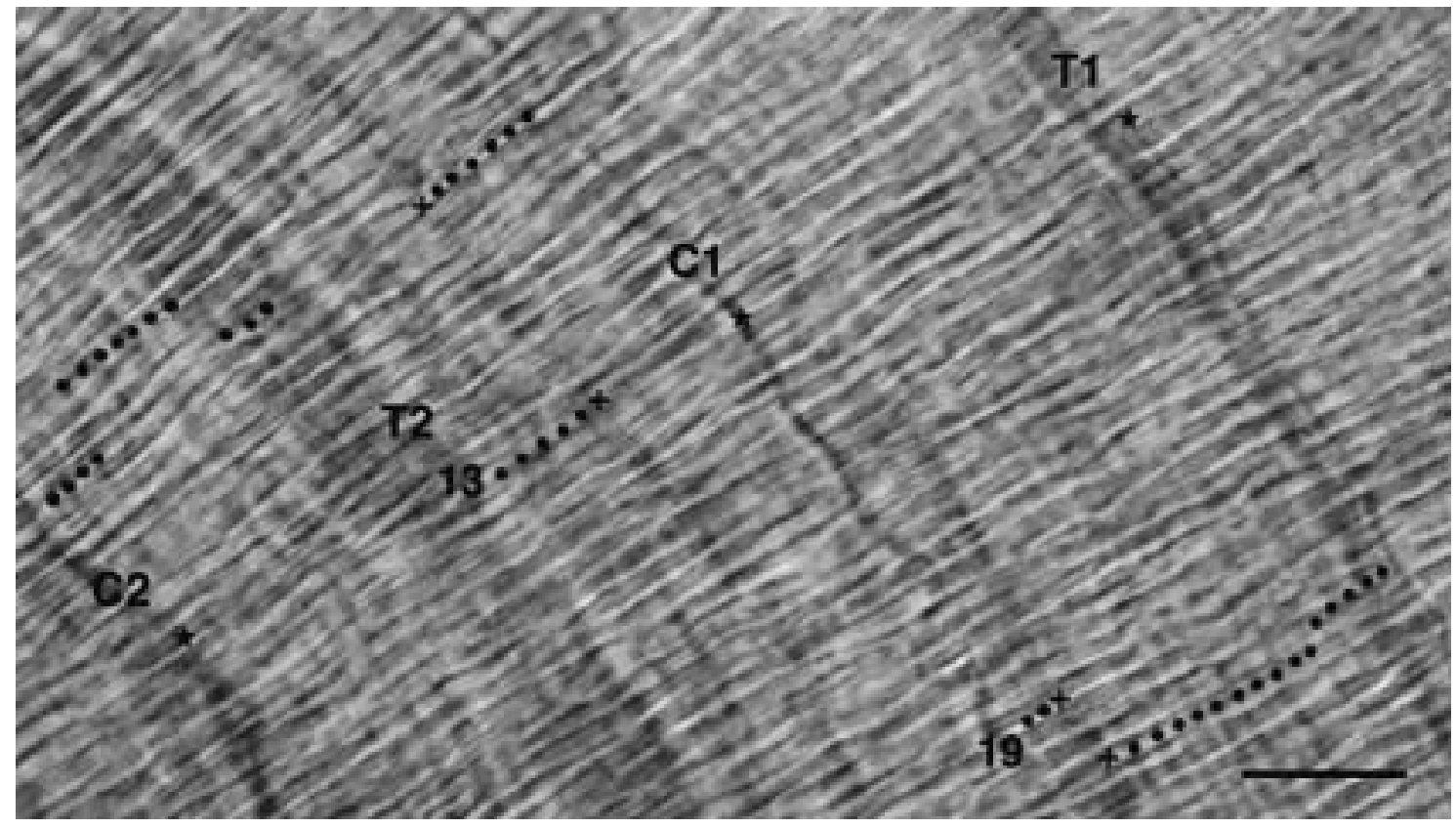

Fig. 4. Higher magnification of photomicrograph of Bodian-stained specimen obtained from sample number VI. The injection history of this sample shows 19-13-15 day intervals. Minute lines cross at right angles to dentinal canals. The number of faintly stained minute regular lines (dot) between successive deeply stained lines (star) is equivalent to that of days between successive injections. Scale bar $=50 \mu \mathrm{m}$. Dot: minute increment. Cross: different point on the same increment. T1: labeled line by the first tetracycline injection, $\mathrm{C} 1$ : labeled line by the first calcein injection, T2: labeled line by the second tetracycline injection, $\mathrm{C} 2$ : labeled line by the second calcein injection. 
13 to 19 increments were present, equivalent to the number of days between the two successive fluorochrome injections (Fig. 4).

The distinct, faintly stained incremental lines began to appear clearly at 200-300 $\mu \mathrm{m}$ inward from the enamel-dentine junction (EDJ), spacing about $5 \mu \mathrm{m}$ interval (Fig. 5). At the inner side of the dentine, the layers were narrowed to less than $2 \mu \mathrm{m}$, gradually disappearing near the pulp cavity.

\section{DISCUSSION}

The deeply stained lines observed in Bodian-stained sections were very similar in appearance to the fluorescent lines in which the deep staining was presumably caused by the administration of fluorochromes in the same manner as seen in the human dentine [17]. It is surmised that $\mathrm{Ca}^{++}$combined with tetracycline was lost by decalcification and instead, $\mathrm{Ag}^{+}$was combined with it [17]. Calcein lines were also inferred to be deeply stained by Bodian's silver technique in a similar manner. In order to analyze the periodicity of increments, a comparison between the chronologically labeled lines and the incremental lines is needed. By this comparison, it was founded that the former occurred simultaneously with the latter on the Bodian-stained specimen. The number of the minute incremental lines existing between successive labeled lines was in accord with that of days between injection-times, suggesting that these lines reflect the daily periodicity in the sika deer dentine.

In the rat, circadian rhythms in the collagen-synthetic and secretory activities of odontoblasts have been found, and these activities might be responsible for the circadian increments in the dentine [11]. It is well-known that dentinogenesis is affected by humoral factors such as growth hormone [3] and parathyroid hormone [19], which are themselves under a strict circadian control. In addition, it has been demonstrated that the suprachiasmatic nucles (SCN) plays an important role in generating circadian dentine increments [12]. Since the dentine incremental lines probably reflect the presence of an endogenous oscillatory mechanism, the circadian rhythm of dentine increments may persist with the neglect of some environmental factors.

From the observations in the present study, it is suggested that the day-age estimation of the sika deer can be conducted with accuracy by using the circadian increments of dentine. The vital labeling intensity may have a close relation to the formation rate of the structure [11]. Since the fluorescencelabeled lines in the ground sections showed a reduced intensity and a narrowed interval toward the pulp cavity, it is suggested that the dentine formation rate is decreased toward the pulp cavity. To estimate age, all the increments from birth to death must be counted. However, in the present study, the daily increments were not distinctly visible near the pulp cavity. If the incremental lines disappear according to the reduction of dentine growth rate, the permanent teeth rather than the deciduous teeth should be investigated in terms of dentine deposition rate. The identification of the

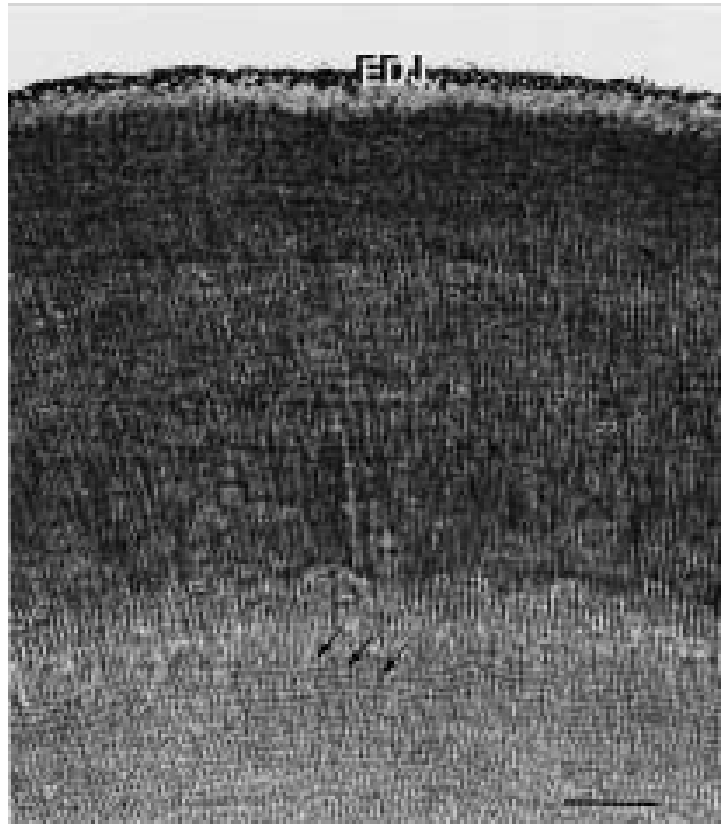

Fig. 5. Outer side of the dentine (Bodian-stained specimen). Incremental lines begin to appear clearly (arrows) at approximately $250 \mu \mathrm{m}$ inward from the enamel-dentine junction. Incremental lines appear repeatedly at approximately $5 \mu \mathrm{m}$ intervals. Scale bar $=50 \mu \mathrm{m}$. EDJ: enamel-dentine junction.

neonatal line is also essential for age estimation. The neonatal line is thought to represent the physiological trauma of birth [14]. Since this line is formed from necessity at a birth, it is useful for assessing age by observing its dental microstructure [5].

In the present study both fluorochromes, oxytetracycline and calcein, constructed clear fluorescent lines in the ground sections. Oxytetracycline-labeling was more diffuse than calcein-labeling, though the dose level of oxytetracycline was regarded as appropriate or even lower in regard to its fluorescent intensity. Thus, both oxytetracycline and calcein are thought to be useful as time markers in the sika deer dentine, but calcein seems to be more valuable with respect to distinctness. However, by our empirical successive injections of one marker alone, some difficulties of interpretation were occasionally encountered at the site of low growth rate when often overlapping of successive labeled lines took place. To avoid such difficulties, the alternate use of two different markers may be helpful.

ACKNOWLEDGEMENTS. We are grateful to the members of the town office of Ashoro District for providing samples. We also thank Dr. S. Yoshida and Miss N. Ushijima for their technical consultation and useful comments. We thank many faculty members and students of Hokkaido University and of Kyushu University Forests in Hokkaido for supporting our research. 


\section{REFERENCES}

1. Erickson, G. M. 1996. Daily deposition of the dentine in juvenile alligator and assessment of tooth replacement rates using incremental line counts. J. Morphol. 228: 189-194.

2. Grue, H. and Jensen, B. 1979. Review of the formation of incremental lines in tooth cementum of terrestrial mammals. Dan. Rev. Game Biol. 11: 1-48.

3. Hansson, L. I., Stenstrm, A. and Thorngren, K. G. 1978. Effect of pituitary hormones on dentin production in maxillary incisors in the rat. Scand. J. Dent. Res. 86: 80-86.

4. Hirakawa, H. and Kaji, K. 1998. The status of sika deer management in various parts of Japan. Mammalian Science (Honyuurui Kagaku) 38: 299-230 (in Japanese).

5. Huda, T. F. J. and Bowman, J. E. 1995. Age determination from dental microstructure in juveniles. Am. J. Phys. Anthropol. 97: 135-150.

6. Keio University, School of Medicine Department of Pathology. 1986. How to make histopathological specimens (Byouri soshiki hyouhon no tukurikata). 6th ed. Igakushoin, Tokyo (in Japanese).

7. Klevezal, G. A. 1996. Recording Structures of Mammals. Determination of Age and Reconstruction of Life History. A. A. Balkema. Brookfield.

8. Klevezal, G. A. and Mina, M.V. 1990. Daily layers and hibernation marks in incisor dentin of Sicista pesudonapaea and some biological remarks. Acta Thriol. 35: 345-356.

9. Myric, A. C. 1984. Time significance of layering in some mammalian hard tissues and its application in population studies. Acta Zool. Fenn. 171: 217-220.

10. Okada, M. 1943. Hard tissue of animal body. pp. 26-31. In:
Shanghai Evening Post, Medical Edition of September.

11. Ohno, K. 1978. On the factors influence the pattern of tetracycline labeling of developing hard tissues. Jpn. J. Oral Biol. 20: 252-269 (in Japanese).

12. Ohtsuka, M., Saeki, S., Igarashi, K. and Shinoda, H. 1998. Circadian rhythms in the incorporation and secretion of $3 \mathrm{H}-$ proline by odontoblasts in relation to incremental line in rat dentine. J. Dent. Res. 77: 1889-1895.

13. Ohtsuka, I. M., Hayashi, H. and Shinoda, H. 2001. Effect of suprachiasmatic nucleus lesion on circadian dentin increment in rats. Am. J. Phys. Regulatory Integrative. Comp. Phys. 280: R1371-R1381.

14. Schour, I. 1936. Neonatal line in enamel and dentin of human deciduous teeth and first permanent molar. J. Am. Dent. Assoc. 23: 1946-1955.

15. Schour, I. and Hoffman, M. M. 1939a. Studies in tooth development I. The 16 microns calcification rhythm in the dentine from fish to man. J. Dent. Res. 18: 91-102.

16. Schour, I. and Hoffman, M. M. 1939b. Studies in tooth development II. The rate of apposition of enamel and dentine in man and other mammals. J. Dent. Res. 18: 161-175.

17. Tanaka, S., Kawasaki, K., Tanaka, R., Shimada, S. and Arai, M. 1997. An application of Bodian's staining to hard tissue. J. Hard Tissue Biol. 6: 70-73 (in Japanese with English abstract).

18. Yilmaz, S., Newman, H. N. and Poole, D. F. G. 1977. Diurnal periodicity of von Ebner growth lines in pig dentine. Arch. Oral Biol. 22: 511-513.

19. Yonaga, T. 1978. Action of parathyroid hormone, with special reference to its anabolic effect on different kinds of tissues in rats. Bull. Tokyo Med. Dent. Univ. 25: 237-258. 